\title{
Revolution of the new environment of the teachers' professional development under the background of the intelligent learning
}

-Exploration of the provincial network-based research and school-based training integration project

\author{
Li Shan ${ }^{1, a}$, Weixin $\mathrm{Hou}^{2, \mathrm{~b}}$, Zhizhong Zhang ${ }^{3, \mathrm{c}}$ \\ 1. Jilin institute of education, Jilin, China \\ 2. Changchun Normal University, Jilin, China \\ 3. The eighth elementary school of auto development area, Jilin, China \\ accshanli@qq.com; b695457431@qq.com; '654239989@qq.com
}

\begin{abstract}
Keywords: Intelligent learning; network-based research; school-based training; teacher's professional development
\end{abstract}

\begin{abstract}
Since the intelligent learning is the realistic demand of the educational development, it can only be effectively satisfied through the revolution of the teachers' professional development environment in order to create the ecological environment that facilitates the teachers to work more smartly. In 2013, according to the Ministry of Education's guidance advice on the deepening of the training pattern reform of the primary and middle school teachers and the comprehensive promotion of the training quality issued by the state government, "the network-based research and the schoolbased training integration project" which facilitates the teachers' professional development in the new era based on the digital environment by means of collaboration is one of the successful modes to implement the spirit of the national policy documents.

The author had hands-on experience and reflected on the revolution of the teachers' professional development environment based on the implementation of the network-based research and the school-based training integration project in Jilin Province. In the essay, the problems existing at the current stage will be analyzed and the suggestions are to be proposed.
\end{abstract}

\section{Introduction}

The "network-based research and the school-based training integration project" ("The Project" for short) is a national training project under the guidance of Ministry of Education's guidance advice on the deepening of the training pattern reform of the primary and middle school teachers and the comprehensive promotion of the training quality issued by the state government. The Project is an optimized design targeting the apparent problems in the teacher training such as weak pertinence, generalization of the content, monotonous modes and weak quality control by taking full advantage of the internet. It is an innovatively smart teacher training and learning pattern with which a brand new smart ecological environment is to be created for the teacher's professional development. In such an environment, the teachers are encouraged to release their potentials of the education wisdom so as to satisfy the realistic demand of the intelligent learning.

\section{Research Design \& Methods}

The Project is to be promoted within the province by the Project Office of Jilin College of education under the guidance of National Center for Educational Technology (NCET)with inservice teacher's education network of the national primary and middle school. The Project involves the primary school and the junior middle school teachers of the following courses: 9 courses in the primary school: Morality and life (society), Chinese, Mathematics, English, Sciences, Music education, Physical education, Fine arts and Comprehensive practice; 13 course in the junior middle school: Ideology and morality, Chinese , Mathematics, English, Physics, Chemistry, Biology, History, Geography, Music education, Physical education, Fine arts and Comprehensive practice. 
The Project Office of Jilin assigned the Project principal for the province-level project to promote the in-depth cooperation with in-service teacher's education network of the national primary and middle school and organize the implementation of the Project within the province. The three counties such as Huadian, Yongji and Changling were selected to participate into the demonstration project.

The school-based training in the Project is not limited to the schools and organization based on the administrative division. In fact, integration of urban and rural area and inter-school cooperation are advocated. In principle, the key schools should assist the schools in the rural areas to conduct the research activity in form of school league and partner school. About 49 schools in the province participated in the Project; 145 network-based teaching and research groups as well as 104 cooperative groups have been established; all in all, 2237 have registered on the network platform.

The promotion of the Project within the province focuses on the design of the role of the personnel. In the county areas, the instructors and researches on disciplines were re-defined in order to extend and shift their functions to the network-based research platform. Based on the number of the faculty and the background of the teachers, the subject research team and cooperative team were established in the campus and the colleges, selecting the directors for the teams. Therefore, a professional teaching and research channel made up of the instructors and researchers, research team leader director, cooperative team director and team members has been established. The channel is free from the administrative intervention of the school. The operation key points of the provincial principals are also transferred from the remote training supervision to the implementation and supervision of the research activities: On one hand, the network-based research mechanism should be established in the counties and districts with the training department of the continuation school as the specific business implementation sector. Thus the management of the instructors and researchers as well as the regulation of the research performance will be conducted. On the other hand, the Project emphasizes the positioning, implementation process of the network-based research, how to integrate it with the school-based training and the key links of the Project. The connotation of the research theme, orientation of the research and the performance of the research process are also attached importance to in the Project.

\section{Results}

After one year practice, the promotion of the Project in Jilin made brand new breakthroughs in the teacher training field and the teacher's professional development environment

Reform 1: establishing the generative learning environment to facilitate the transformation of the contradictions between learning and working.

The contradiction between working and learning is always the issue the teachers have to be confronted with in the in-service learning. For a long period of time, it has been the common practice that the teachers learn and reflect before practicing in teaching whether when they attend the expert seminars and the case study training or the participatory and immersion training, or the web-based remote training and face-to-face training. As a result, the in-service learning of teachers is divided to two steps of learning and working, and learning always goes first. However, after years and rounds of such a kind of teacher trainings, we must admit that the teachers may be impressed deeply during the training, but little changes have actually taken place in their teaching practice. The training process that separates learning and working eventually causes the contradiction between learning and working. The effect of the teaching practice is also proved to be not satisfactory.

Changes are hard for people and so is the course of growing up for teachers who must experience a shift from reflections to actions. After the teachers come back to the familiar teaching environment from the training, everything seems to regress. It is therefore not easy to apply what they have acquired in the training to the practice, for as for most of the people, the transition from cognition to action is not a spontaneous process but requires the guidance of the transition method and establishment of the transition environment. Therefore, to resolve the contradiction between 
learning and working, we need to change the environment and pattern of the in-service learning of the teachers and integrate learning and working.

The Project constructed a practice model of “integration of learning with working" from the perspective of design and the generative learning environment in order to resolve effectively the contradiction between learning and working. Since the research space highlights the generativity, in the Project, the network not only provides abundant course for the teachers but also various theme activities around the key issues in the teacher's professional development. Under the guidance of the whole set of the research tasks, the teachers may be more clear about the field and direction of their professional development. Then the learning state of the teachers would change: they will become the subject of learning. They will focus on what they accept but what they can generate; they will become the creators of thoughts and wisdom and the trainers that output their ideas and cooperate with each other. We will strive to integrate the learning process with the teaching practice in order that the teachers can learn from teaching and teach from what they have learnt.

Reform 2: Promoting the transformation of the modern teaching and research patterns

The implementation of the Project brings changes to the patterns of teaching and research and facilitates the transformation from the traditional teaching and research to the modern one. All the activities such as lesson preparation are conducted both online and offline: The teachers accomplish the teaching and research process online and discuss about the issues about teaching offline with teaching design as the core.

Firstly, we should emphasize the transition from the teaching-oriented to the learning-oriented to conduct various class teaching patterns suitable for the school such as "learning goes before teaching; classroom training" and " 3 links and 5 steps". In the original teaching framework, it is tough to change from the teaching-oriented to the learning-oriented because the concept is undoubtedly good but in practice, it would be rather difficult. Through the in-depth implementation of the Project, in the smart ecological environment, the teachers come to realize that they should firstly make profound analysis on the standard requirement of the course and re-refresh the understanding about the disciplinary knowledge to drop out the traditional teaching approach only focusing on the knowledge point so as to realize the transformation of the teaching method. The learning-oriented approach would be more reflected on the development of the students' high order thinking. By far, teachers have conducted in-depth reflections and practice in many aspects such as the students' participatory activity design technology, the question design and home assignments.

Secondly, we should consider the teaching application of the information technology in class the important content in the school-based training. To avoid any blind application of the information technology, we should firstly identify where the information technology is required as well as the teaching requirement and then seek for the solutions from the information technology. Therefore, the teaching and research groups and cooperative groups should select and produce the course resource together in order to make a "resource package" for the school-based training. Through the teaching and research activities, for example, different structures for the same topic, the teachers can share the resource in the research and teaching space and discus about how to apply the resources.

Thirdly, we should shift our focus from the knowledge instruction to the human development, particularly the cultivation of the cooperation awareness in class. With the joint efforts of the teachers and students in the long run, the cooperative learning will demonstrate its advantages. Only when the teachers experience cooperation and understand profoundly the course of cooperation can they instruct the students to cooperate in the learning activities effectively.

Reform 3: Establishing the research cooperative team and re-shaping the social connections of teachers

For a long period of time, the path of the teacher's professional development is too administrative; the professional environment of the teachers is full of competition and the assessment for teachers' performance is one-fold. Therefore, the career wellbeing and sense of the identity of the professional development are rather low, which would easily affect the working enthusiasm of the 
teachings for their work and interior drive for their professional development and thus restrain the generation of the educational wisdom of a multitude of the grassroots teachers.

By the combination of the online and offline spaces, the Project cultivates many practice teams in form of research groups and cooperative groups and establishes the collaborative relations among the teachers. The teachers can put their time, energy and wisdom on the more important, more valuable and more significant tasks. And they can solve complex teaching problems by cooperation with the professional wisdom and potentials of every teacher opened and triggered.

The research cooperative team will enable the academic performance, teaching and research capacity and educational capacity to be recognized. Their educational ideas can be effectively transmitted and they can discuss about any puzzles in teaching with their peers and experts. The teachers will be more enthusiastic for learning and working; the teaching and research would be no longer based on the individuals but a team. In the other word, the process would be brainstorming.

To sum up, the network-based research and the school-based training integration project which makes difference to the traditional learning approach of remote training for the teachers establishes the cooperative and digitalized ecological environment characterized by "integration of learning with working” in which the teachers' wisdom and potentials in teaching will be triggered. During the design and implementation process of the Project, we have made beneficial attempts about the reform of the teacher's professional development environment under the background of the intelligent learning and constructed a brand new teacher's professional development practice model in order to facilitate the transformation of the modern teaching and research patterns and the reshaping of the social connections of the teachers and to open the path for the cultivation and progress of the intelligent-type professional teachers.

\section{Thinking on Development \& Construction}

During the promotion of the Project in the province, we will integrate the web-based research platform and the offline practical teaching and research as well as the teaching activities. We were confronted with some challenges in the course but we also explored the actual requirements of the teachers for training and teaching. Therefore, we made the following proposals in terms of the design and implementation of the Project:

1. Transforming the online course resource patterns

Nowadays, most of the web-based training videos are the expert-report videos which are lack of the extraction of the logic ideas and opinions and the concentration on the specific links in teaching; in terms of the form of the videos, the duration is too long and the speaking speed and pace is slow and verbose; Most of them seem to be too general and extensive; the case study is complete but lack of the presentation of the essential problems in education.

With the concepts such as MOOC, Micro-class and Micro-video are widely known in the educational field, changes must take place in the learning method of the teachers. Everyone has limited time and energy. It is the same for the teachers. It is thus suggested that the Micro-video can be applied to conduct the trainings with pertinence. We should provide more typical cases to enlighten the teachers to think profoundly and guide them to evolve to the high-order way of thinking so that the teachers can guide the students to the high order.

2. Enhancing the advanced wisdom guidance of the specialists

At present, there are some cognitive limitations in the grassroots teaching and research ideology and methodology. For example, there is a lack of the instruction of the researchers and professional personnel in the county-level, district-level or school-based training activities. As the part of the Project, we will provide experts in various subjects to instruct and answer inquiries regularly online, but the supply-demand relationship is still not balanced.

On one hand, the wisdom of many experts and specialists should be absorbed in the web-based research ecological environment in order to change the mechanic knowledge transmission chain during the previous training process of the teachers to the open wisdom space for anyone to have access to. Meanwhile, the layer-by-layer information delivery channel should be simplified to broaden the vision of the grassroots educators and fast improve the ideological capacity of the 
teachers to avoid the low-level and static research and learning as well as the superficial discussion and solutions. With the big wisdom field created on the network, the wisdom of the teachers can be aroused, which helps the teachers transit their ideology and wisdom to the educational practice and behaviors more effectively and eventually benefit the students who can learn smartly.

On the other hand, we need to replace the passive reception of the report-like instillation and instructions. Instead, we need to integrate the lectures and the reports given by the experts with the research practice as well as the entire process of class preparation. We are to conduct research activities online, completing the instruction, evaluation and discussion. Offline, the teaching and research activities can be conducted synchronically with the school-based training as the main form in order to gather higher, more profound and more abundant teaching wisdom.

To sum up, in the ecological system integrated with the network-based research and school-based training, what we do not need is preaching. Instead, we advocate the discussion about how to make sense with collective intelligence by exploring the realistic problems in practice in order to find the solutions and strategies for group discussion. Then we can summarize, conclude and induce during the discussion and stimulate the front-line teachers to reflect and seek for the solutions. By that means the capacity of the teachers can be promoted in the real sense and it can truly make some differences to the teaching practice.

3. Establishing the research team culture

The establishment of the learning team and the team culture has been a concern for a long time in the research and training field of the teachers. During the experimental and participatory trainings, the grouping and establishing the team culture have been a common practice. However, the teams are just temporary and the effect is thus not lasting. After the training is over, the teams just disappear, which means that the teachers need urgently a normalized team environment. Therefore, in the ecological environment created by the Project, we find it important to establish relatively stable, feasible and sustainable team or organizational structure.

Currently, the collaboration practice among the teachers in the network-based research environment is still superficial, at the level of the individual practice. No powerful subject collaborative teams have taken shape. As we known, the team cultural style and the design of the division of labor in the organization would be the highlight and concern in the future studies. In particular, under the background of the intelligent learning, the significance of the smart education, smart class, teacher's research cooperative teams and the establishment of the team culture would be even more prominent.

\section{References}

[1] Bian Zhixian, Xiong Caiping, Yang Wenzheng et al. The teaching model and simulation that integrates the web-based teaching and research into the credit assessment system for the teacher's in-service education. [J]. China Educational Technology, 2013.

[2] Norbert. M Seal, Sonny Dykstra. International outlook of the curriculum, planning and process in the teaching design [M]. Beijing: Education Science Publishing House, 2009.

[3] The ministry of Education of People's Republic of China. Standard for the information technology application of the primary and middle school teachers (trial). [EB/OL] . http://www.moe.gov.cn, 2014 .

[4] Gu Lingyuan. Teachers and their instructors from the perspective of class. [J]. Courses, Textbooks, Teaching Methods, 2014.

[5] Curtis. J. Bunker. It’s an open world. [M]. Shanghai: ECNU Press, 2013. 